\title{
Why prevention instead of cure of chronic diseases?
}

\begin{abstract}
This article explains that since the onset of chronic diseases is slow ('chronic' means 'slow') and the cure of chronic diseases is also slow, difficult (it requires a series of 14 to 20 daily acupuncture treatments) and unpredictable (the series might need to be repeated), prevention of chronic diseases should be our goal. The slow onset of chronic diseases leaves plenty of time for this. When pulse diagnosis detects early deviations from norm (before the symptoms of the disease had appeared), one acupuncture treatment is usually enough to prevent the disease. Thus, the chronic diseases need early preventive cure, which is fast and easy, and there is no element of unpredictability.
\end{abstract}

Keywords: Acupuncture; Cchronic diseases, Cure of chronic diseases; Pulse diagnosis; Prevention of chronic diseases
Volume II Issue I - 2018

M Kuman

Holistic Research Institute, USA

Correspondence: M Kuman, Holistic Research Institute, 1414 Barcelona Dr., Knoxville, TN 37923, USA,

Email holisticarel@gmail.com

Received: January 20, 2018 | Published: January 24, 2018

\section{Acupuncture is now used to cure diseases}

While in ancient China acupuncture was basically used for prevention, now acupuncture is mainly used to cure chronic diseases. According to ancient texts, each attempt to cure a chronic disease after its onset is an attempt to make guns when the enemy is already in your yard - it is too late. ${ }^{1}$ No wonder we are not successful in the cure of chronic diseases - every third elder American (over age of 70) suffers from one or more chronic diseases.

Both chronic diseases and cancer result from prolonged stress, which means either one strong prolonged stressor, or a series of mild stressors (daily hassles) influencing the body through relatively short time intervals, which allows their nonspecific effects on the body to accumulate until the ultimate level of stress, which the genetically inherited weak organ can tolerate, had been reached. This results in chronic disease or cancer of the genetically weak organ (genetic predisposition determines chronic disease or cancer would be developed). If so, a simple idea comes to mind. Since accumulation of stress brought the pathology, we should be able to cure this pathology by using the stimulation effect of a series of mild stressors, such as daily acupuncture treatments. Then the long-lasting stimulation effect of each treatment (two to three days) ${ }^{2}$ will allow accumulation of the effect of each treatment, which will eventually lead to cure of the chronic disease.

Therefore, since chronic diseases and cancer are diseases induced by strong or prolong stress, a series of mild stressors, which stimulate, could be used to cure them. In principle any mild stressor can be used for cure: needles (classic acupuncture), moxa or infrared light, acupressure, mild laser, electric current, magnets, garlic, etc.

It works on the principle a wedge drives out wedge. The essential thing here is to treat every single day until the disease is cured because only daily treatments will allow accumulation of the stimulation effect of each treatment. Since any stressor could cure chronic disease or cancer, future acupuncture would probably not use needles and would be basically used for prevention (for details see the author's book ${ }^{3}$ ).

\section{Why prevention instead of cure of chronic diseases?}

We should monitor the changes that precede the chronic disease.
In ancient China, every patient was paying an annual fee and visiting his acupuncturist four times a year for a regular check up with pulse diagnosis. If small deviations from norm were noticed somewhere, they were balanced with acupuncture and usually one acupuncture treatment was enough to restore the balance and bring back the health. If the patent happened to get sick, the acupuncturist was supposed to treat him for free because he didn't do his preventive job properly. In this way, it was in the doctors' interest to keep the patient healthy (see the author's book: Kuman M, Modern Aspects of Ancient Acupuncture, Chapter 5). Pulse diagnosis is still used in the East - in China, Japan, Korea, etc.

The pulses of the organs on the surface of the skin are measured and the pulses of neck, arms and legs compared, which allows discovering of slight deviations from norm. In this very early stage of the chronic disease, when symptoms are not experienced yet, one acupuncture treatment is usually enough to prevent the oncoming disease.

Pulse diagnosis is not popular in the western world, but equipment adequate to pulse diagnosis was built and successfully used in England. ${ }^{4}$ The pulses of different organs were measured, recorded, and compared. This allowed very early diagnosis of chronic diseases a few years before the symptoms of the disease would appear.

As stated in the author's book: Maria Kuman, What Everybody Ought to Know about Chronic Pain, Chronic Diseases, and Cancer, ${ }^{2}$ cancer is also a slow disease, if the weakening of the cellular immune system by stress is considered. The cure of cancer is also slow, difficult, and unpredictable, just like the cure of chronic diseases is. Just when we claim victory over it, it reappears. Therefore, cancer also requires preventive measures. The slow onset of both chronic diseases and cancer leaves plenty of time to try to intervene and restore the balance of the body before the pathological state is reached (for details see the author's book).

\section{Signal points to be treated for prevention of chronic diseases}

The so-called 'signal points' are acupuncture points, which signal oncoming pathology. They are painful or tender to the touch. The table below lists the 'signal points' on the back, which need to be checked for tenderness for early diagnosis of oncoming pathology of different organs. ${ }^{5}$ 


\begin{tabular}{llllllllllll}
\hline LU & PC & HT & LR & GB & SP & ST & TE & KI & LI & SI & BL \\
BL13 & BL14 & BLI5 & BLI8 & BLI9 & BL20 & BL21 & BL22 & BL23 & BL25 & BL27 & BL28 \\
BL42 & BL43 & BL44 & BL47 & BL48 & BL49 & BL50 & BL5I & BL52 & & & BL53 \\
\hline
\end{tabular}

There are also other points that signal oncoming pathology. For example, ST27 is the diagnostic point for large-intestinal troubles according to Dr. Manaka et al. ${ }^{5}$ If point ST27 is painful or tender to the touch, troubles in the large intestines could be expected. Treatment of the 'signal points' together with other major points of the organ can prevent chronic disease or cancer with one or two treatments.

\section{Acknowledgments}

None.

\section{Conflict of interest}

None.

\section{References}

1. Kuman M. Modern Aspects of Ancient Acupuncture. $2^{\text {nd }}$ ed, USA: Health and Happiness Books; 2012.

2. Waldman AB. Actualnie Problemi Stressa (Russ). Kishinev: Moldova; 1976.

3. Kuman M. What Everybody Ought to Know about Chronic Pain, Chronic Diseases, and Cancer? $2^{\text {nd }}$ ed, USA: Health and Happiness Books; 1997.

4. Kenyon J. Modern Techniques of Acupuncture. $2^{\text {nd }}$ ed. England: Thorsons Publishers Limited; 1983.

5. Manaka Y (1995) Chasing the Dragon's Tail. Paradigm Publications, USA. 